COMMENT. This study demonstrates an increased incidence of CP and DD/MR for children born moderately preterm. Late preterm infants should be examined neurologically and followed through early childhood to exclude or treat associated developmental learning disabilities. The risk of adverse neurodevelopmental delay or $\mathrm{CP}$ decreases with increasing gestational age.

\title{
MARKERS OF DEVELOPMENTAL SYNESTHESIA IN CHILDHOOD
}

The prevalence and development of grapheme-color synesthesia in children in the UK and US and its progression in longitudinal testing over 12 months (from ages 6/7 to 7/8) were studied at the Department of Psychology, University of Edinburgh, Scotland, UK. Individuals with synesthesia have the ability to merge sensory and/or cognitive functions. Everyday activities such as reading may trigger extraordinary experiences, eg colors or tastes. These atypical sensations arise spontaneously during development. In adults with synesthesia, a triggering stimulus (inducer) consistently triggers the same concurrent experience over time, referred to as the behavioral hallmark of synesthesia. Synesthetic experiences have anatomic and genetic roots. The average UK primary school has 2-3 grapheme-color synesthetes, and the average US primary school has 5. Synesthetic associations (eg. the letter "a"=carmine red) develop from chaotic pairings into a system of fixed cogno-sensory responses over time. Synesthesia has benefits and costs for the individual. Children who experience tastes from words read or spoken have difficulties in maintaining attention when reading, while grapheme-color synesthetes show superior color and digit memory. (Simner J, Harrold J, Creed H, Monro L, Foulkes L. Early detection of markers for synaesthesia in childhood populations. Brain Jan 2009;132:57-64). (Respond: Julia Simner, Department of Psychology, University of Edinburgh, 7 George Square, Edinburgh, EH8 9JZ, UK. E-mail: j.simner@ed.ac.uk).

COMMENT. In Webster, synesthesia is defined as a concomitant sensation, a subjective sensation or image of a sense (as of color) other than the one (as of sound) being stimulated. DeJong RN (The Neurologic Examination. $3^{\text {rd }}$ ed, New York, Hoeber, 1967) uses the terms synesthesia, together with allachesthesia, allesthesia, and allochiria when the sensation of touch is experienced at a site remote from the point of stimulation. Neurologists have been aware of the phenomenon of synesthesia but, except for the neuropathologist, Yakovlev PI (J Nerv Ment Dis 1948;107:313-335), do not appear to acknowledge it as "an involuntary physical experience" that is real in the mind of the subject affected. Cytowic RE (Psych 1995;2(10) in a review of synesthesia describes the sensation as a cross-modal association, a familial trait, more common in females and non-right handed subjects. The neurological examination is usually normal. Synesthesia is considered a left hemisphere function, involving the hippocampus. Behavioral correlates include superior memory and weakened math and spatial perception. A recent resurgence in interest in synesthesia is lead by the field of neuropsychology and cognitive science. A PubMed search uncovers 7 references on synesthesia, none authored by a neurologist. Usually regarded as rare, two studies estimate that $5 \%$ of the population may experience at least one type of synesthesia. Epileptologists might be interested to research the prevalence of synesthesia in patients with temporal lobe seizures. 